\title{
Clinical and neurophysiological patterns of impairments to emotion attention and empathy in multiple sclerosis
}

\author{
Michael Adamaszek ${ }^{1,2, *}$, Stefan Krüger ${ }^{3,4}$, Christof Kessler ${ }^{2}$, Norbert Hosten ${ }^{5}$, Alfons Hamm ${ }^{4}$ \\ ${ }^{1}$ Department of Clinical and Cognitive Neurorehabilitation, 93413 Klinik Bavaria Kreischa, Germany \\ ${ }^{2}$ Department of Neurology, University of Greifswald, 17489 Greifswald, Mecklenburg-Vorpommern, Germany \\ ${ }^{3}$ Ambulance of Psychotherapy, 14547 Beelitz, Germany \\ ${ }^{4}$ Institute of Physiological and Clinical Psychology, University of Creifswald, 17489 Greifswald, Mecklenburg-Vorpommern, Germany \\ ${ }^{5}$ Department of Diagnostic Radiology and Neuroradiology, University of Greifswald, 17489 Greifswald, Mecklenburg-Vorpommern, Germany \\ *Correspondence: michael.adamaszek@klinik-bavaria.de (Michael Adamaszek)
}

\section{DOI:10.31083/j.jin2101007}

This is an open access article under the CC BY 4.0 license (https://creativecommons.org/licenses/by/4.0/).

Submitted: 11 March 2021 Revised: 9 April 2021 Accepted: 23 June 2021 Published: 28 January 2022

Patients suffering from multiple sclerosis experience various cognitive and affective impairments, resulting in a negative impact on social behavior and personal independence to differing degrees. According to these often clinically subtle but conflicting cognitiveaffective impairments, recordings of these socially relevant issues are still of demand to stratifying clinical and social support in a sophisticated way. Therefore, we studied specific cognitive and affective capacities in eleven patients with a predominant relapsing-remitting type of multiple sclerosis by applying paradigms of event-related potentials and a well-selected neuropsychological test protocol. Thus far, distinct cognitive disturbances of executive and attentional domains and the Wechsler Memory Test's four memory indices were found in multiple sclerosis patients. Concerning affective domains, patients showed discrete impairments of affect discrimination and affected naming as proved by specific testing (Tuebinger Affect Battery). Neurophysiologically, event-related potentials recordings in multiple sclerosis patients, were associated with decreased implicit emotion processing to cues of different emotion arousal at the early processing stage depending on attentional capacities and alterations of implicit emotion modulation at late processing stages. These clinical neurophysiological and neuropsychological data were correlated in part to quantitative magnetic resonance imaging brain lesions. Summarizing our data, our data indicate certain neurocognitive and neuroaffective dysfunctions in patients with multiple sclerosis, thus highlighting the validity of sensitive recording of less apparent neurologic disturbances in multiple sclerosis for optimizing the individual care management in patients.

\section{Keywords}

Multiple sclerosis; Emotion attention; Emotion empathy; Event-related potentials

\section{Introduction}

Approximately $70 \%$ of patients with multiple sclerosis (MS) at all stages and in all subtypes of the disease exhibit various cognitive dysfunctions during their illness [1-7]. Neuropsychological tests consistently reveal impairments to sev- eral cognitive domains such as episodic and working memory, attention, executive functioning, and information processing speed as the significant areas [8-13]. Besides these cognitive impairments, patients suffering from MS are also at particular risk for affective disturbances such as perceiving and recognizing emotion with their sequelae to social interaction and affective functioning [14]. In addition, functional impairments, including disorders of affect and behavior, anxiety disorders, and substantial personality changes, including irritability, emotional lability, and apathy, account for up to $60 \%$ of MS patients for another leading contributor to morbidity and mortality [15]. In MS, disconnections in the frontal-subcortical brain tracts, known to also be involved in processing emotion signals, are observed [16]. However, the observed impairments in emotion processing in MS with failures incorrect identification of emotions might depend partly on the necessities of the functional integrity of a number of cognitive domains, including working memory, visual attention, visuospatial perception, and executive functions often compromised in MS [14]. In particular, impaired attention, executive function and working memory might be a matter of reduced process quality at the prefrontal cortex sites in forwarding cues of the emotional content of the inner and/or outer environment, so studying affective impairments at one hand, but also the consideration of possible confounding high order cognitive disturbances at the other hand, seems to be reasonable for implementing cognitive domains in studying emotion processing in MS, guiding a comprehensive approach in disentangling the features of affective impairments in MS.

Morphological and tractographical MRI studies in patients with MS suggested morphological changes of the white and gray matter within frontal, temporal and parietal lobes, suggesting disturbed structural integrity of the responsible frontal-parietal networks according to their functions along substantive cortical and subcortical pathways onto the result- 
ing affective, but also cognitive impairments [17-19]. As far as these structural lesions and subsequent brain atrophy are well recognized as causing affective and cognitive decline [20], still circumscribed lesions as putative for distinct cognitive or affective disturbances have been criticized for several reasons. Nonetheless, some studies have been able to find significant correlations between clinical impairments of cognitive and/or affective domains and lesions located, for instance, in structures of the frontal lobes [21], the limbic system [22] and the left arcuatus fasciculatus [23], are robustly identified in association with the observed high order domains of cognitive and/or emotion processing.

Beyond the structural analyses provided by cranial MRI, investigating the neurophysiological underpinnings of cognitive and/or affective impairments in MS would be of specific interest, particularly for capturing the affective impairments along their different time distribution lines. Using suitable paradigms could support this to evoke specific eventrelated potentials (ERP), but there are rare substantial reports on these issues. Specific investigations using the P300 component have given some interesting information on the neurophysiological background of cognitive impairments in MS [24-30]. In contrast, substantial reports about ERP patterns for emotion processing in MS are a subject of interest, particularly considering the previously conclusive findings of event-related potentials to physiological emotion processing in healthy subjects. The hallmark findings of ERP in emotion processing are early negativity shifts around 200 and $300 \mathrm{~ms}$ post stimulus to pictures with salient emotional contents of different arousal over temporo-occipital areas in healthy humans, indicative of an early facilitated sensory processing of affective cues at the bottom-up of the extended visual system independent of top-down control [31-34], and also indicative of augmented late positive potentials over parietal areas, indicating an intrinsically higher relevance of affective cues additionally on subsequent order stages of processing affective cues [35]. These robust findings along with various studies of healthy populations suggest a fairly conservative neurophysiological feature of emotion processing, which could be compromised by distinct neural lesions of the brain, offering a particular avenue for studying the neurophysiological background of emotion processing in neurological disorders as a sequela of topographically distinct brain lesions [36, 37].

The present study aimed to study this neurophysiological background of emotional disturbances in MS in more detail. Concerning recording early and high order emotion modulation by confined event-related potential paradigms, we hypothesized that patients with MS would show altered ERP of emotive cues of salient scenes at the early and also late processing as representative for affective processing at bottomup and top-down stages, which should emphasize specific neurophysiological patterns as underpinning the clinically described affective impairments in MS patients. As a second hypothesis, impaired discrimination in emotions should also be found clinically, i.e., by testing the recognition of emo- tions in the Tübinger Affekt Batterie (TAB) facial expression, which might support the primary hypothesis of electrophysiological patterns of disturbed affective processing. Concerning probing the prevalence and possible influence of cognitive deficiencies on affective impairments, we applied a broad neuropsychological test battery focusing on attentional, memory and executive functions in our study sample. Another aim of the present study was to identify substantial correlations between the investigated ERP patterns and the clinical and structural data provided by lesion load in cranial MRI, respectively.

\section{Materials and methods \\ 2.1 Patients and controls}

We examined eleven ambulatory patients with a confined relapsing-remitting course of multiple sclerosis (RRMS), whereby three patients met the criteria of an advanced illness stage of the secondary chronic course of MS, according to the criteria of McDonald et al. [38, 39]. The patients have been recruited from institutional care of the department, comprising four male and seven female patients with a median age of 37.36 years. For control, eleven convenient, healthy subjects of the investigator institute surrounding comparable sex, age, and education level were considered without any neurological or psychiatric disorders. Patients enrolled in the study were diagnosed with clinically defined MS by an experienced neurologist according to the McDonald criteria [38, 39]. All the patients were in the course of treatment with diseasemodifying agents, including interferon beta and glatiramer acetate. Exclusion criteria included severe cognitive or affective disorders as its entity according to ICD10, progressive forms of MS, the concomitance of other neurological or systemic disorders, severely decreased visual acuity and hearing loss.

Around clinical neuropsychological and psychophysiological testing, an MRI for further assessments of correlation of obtained data was performed within seven days. Patients were examined clinically with the MSFC (Multiple Sclerosis Functional Composite): walking distance, 9-hole-peg-test, Paced Auditory Serial Addition Test for assessment of overall motor and cognitive performance, and EDSS (Expanded Disability Severity Scale) for the graduation of daily dependency [40]. We informed all patients and controls about the design and aims of the study, and they gave their consent. All subjects performed the neuropsychological test battery and the event-related potentials recordings. The mean performing duration time of neuropsychologic testing and recording of event-related potentials was around three hours. Cranial MRI sessions for each MS patient did not extend over half an hour. The local ethics committee approved the study.

\subsection{Neuropsychological testing}

Patients of MS and controls were tested with the Tübinger Affekt Batterie (TAB) and adapted and validated the German version of the Florida Affect Battery [41]. This battery contains ten subtests, discriminating five different basic 
emotions (happy, sad, angry, anxious, neutral) along visual, acoustic or intermodal (visual-acoustic) processing streams of responsible neural networks. In our study, the subtests 15 (1: facial identity discrimination; 2: facial affect discrimination; 3: facial affect naming; 4: facial affect selection; 5: facial affect matching) were engaged to study the recognition of the basic emotions in facial expressions. Additionally, probing cognitive domains along with attentional, executive and memory functions, we applied the test battery of attention probing (Test of Attentional Performance; TAP) containing five subtests (alertness, neglect, divided attention, covered shifting of attention; incompatibility) [42]. To test executive functions, we used the CKV (Computergestütztes KartensortierVerfahren), a German computerized version of the Wisconsin card sorting test by the computer-served card sorting procedure assessment disturbances of categorial properties [43]. The test evaluates executive functions, such as abstract thinking, development of strategies and playful action. Assessment of special memory capacities was tested by the revised Wechsler Memory Test (WMS-R) [44].

\subsection{Event-related potentials}

Studying the event-related potentials to emotional cues, we engaged computerized video movies made using Adobe ${ }^{\circledR}$ Premiere ${ }^{\circledR}$ software on a G3 Power Macintosh ${ }^{\circledR}$. The videos presented 702 and 699 pictures as continuous streams of images. Videos were presented on a 21 -inch EIZO F77 computer screen located approximately $100 \mathrm{~cm}$ before the subject, without perceivable inter-stimulus intervals $(85 \mathrm{~Hz}$ refresh rate). Brain and ocular scalp potential fields were recorded with a multichannel 129 lead geodesic sensor net (Electrical Geodesics, Inc.), ensuring an evenly distributed sensor layout over the head surface with an intersensor distance of about $28-30 \mathrm{~mm}$. Electrode impedance was kept below $30 \mathrm{k} \Omega$. Data were recorded continuously with the vertex sensor as a reference electrode. The data were online bandpass filtered from $0.01-100 \mathrm{~Hz}$ and sampled at $250 \mathrm{~Hz}$ using Netstation software (5.2, EGI, Oregon, USA) and EGI amplifiers.

Event-related potentials for emotion encoding were recorded after applying a standardized set of paradigms [34, 35]. Colored pictures of the International Affective Picture System (IAPS) were presented to the subjects containing different emotional valences involving enjoyable (e.g., erotica, adventures, and sports) and very unpleasant contents (mutilations, human violence, and animal threat). Furthermore, pictures of a low level of arousal with different emotional valence, either neutral (e.g., household objects, neutral faces), less pleasant (e.g., babies, foods, and family scenes), and less unpleasant materials (e.g., loss, contamination, pollution) were presented [32]. All pictures were depicted in perceptually random sequences in each run of picture presentation. Four experimental conditions, each a constructed movie containing various examples of the IAPS, were administered (see Fig. 1). Conditions 1-3 each presented 702 pictures as a stream with a displaying duration of $333 \mathrm{~ms}$ per image, condition 4 presented 699 pictures with a displaying duration of $1000 \mathrm{~ms}$ per image, drawn from a random order:

(1) Condition 1 was a 'viewing only condition' requesting the participant to fixate on the screen and watch the images, examining visual attention to emotional cues as indexed by an Early Negative Potential (EPN). In the second and third conditions, two different series of pictures of IAPS were displayed in the same manner (primary implicit visual task) but comprised with an explicit cognitive task focusing attention on non-affective stimuli (secondary explicit visual and/or auditory task). (2) In condition 2, a comparable movie was presented as a stream. Still, like an oddball paradigma, each picture was overlaid with vertical or horizontal lines. The patient required to count vertical lines, interspersed in random order and a probability of $20 \%$ of pictures overlaid with vertical lines ('visual secondary cognitive attention'). (3) In condition 3, instead of lines, either a tone of low $(800 \mathrm{~Hz})$ or high $(1000 \mathrm{~Hz})$ frequency was presented with each picture, with the participant required to count high tones which were interspersed in random order and with a probability of $20 \%$ of pictures accompanied with a high tone ('auditory secondary cognitive attention'). Participants were asked after each trial how many targets had been counted. In both conditions (2 and 3), the task-related non-affective stimuli were interspersed in a randomized order in the IAPS picture sequence with a mean lag of 6 IAPS pictures in between (range 2-10 pictures). (4) Condition 4 was similar to condition 1 , but with a more extended presentation of $1000 \mathrm{~ms}$ per picture to examine Late Positive Potential (LPP) ('second viewing only condition'). In this condition, the participant had been requested again to fixate on the presentation screen, as he did in the first trial, to capture early implicit encoding of affective cues (all four trials are depicted in Fig. 1).

Subjects were instructed carefully before each movie presentation, especially to stay fixated on the center of the screen. To minimize effects due to stimulus novelty, the subject was familiarized with the complete picture stimulus set. For all experimental runs, all subjects were informed clearly about the character and the task of each video presentation. Each subject was instructed to maintain focus on the center of the screen in each movie. Every video was followed by a break of approximately 5 minutes, in which the signal quality of the EEG sensors was checked. Afterward, task instructions for each experimental condition were given separately. Each presentation of the video lasted around 4 minutes. The subject room was dimly lit during a presentation of the videos.

\subsection{Event-related potentials: data analysis}

A $40 \mathrm{~Hz}$, low pass filter was applied offline to the continuous EEG data. Stimulus synchronized epochs lasting from $42 \mathrm{~ms}$ before $332 \mathrm{~ms}$ after picture onset for $333 \mathrm{~ms}$ presented pictures, and pictures presented $42 \mathrm{~ms}$ before until $900 \mathrm{~ms}$ after $1000 \mathrm{~ms}$ were extracted. Off-line analysis of event-related potentials was performed by a Matlab-Analyzer package, excluding global artifacts like movements and correction for ocular movements after conversion to an average reference. Individual channel artifacts were detected based on the original 

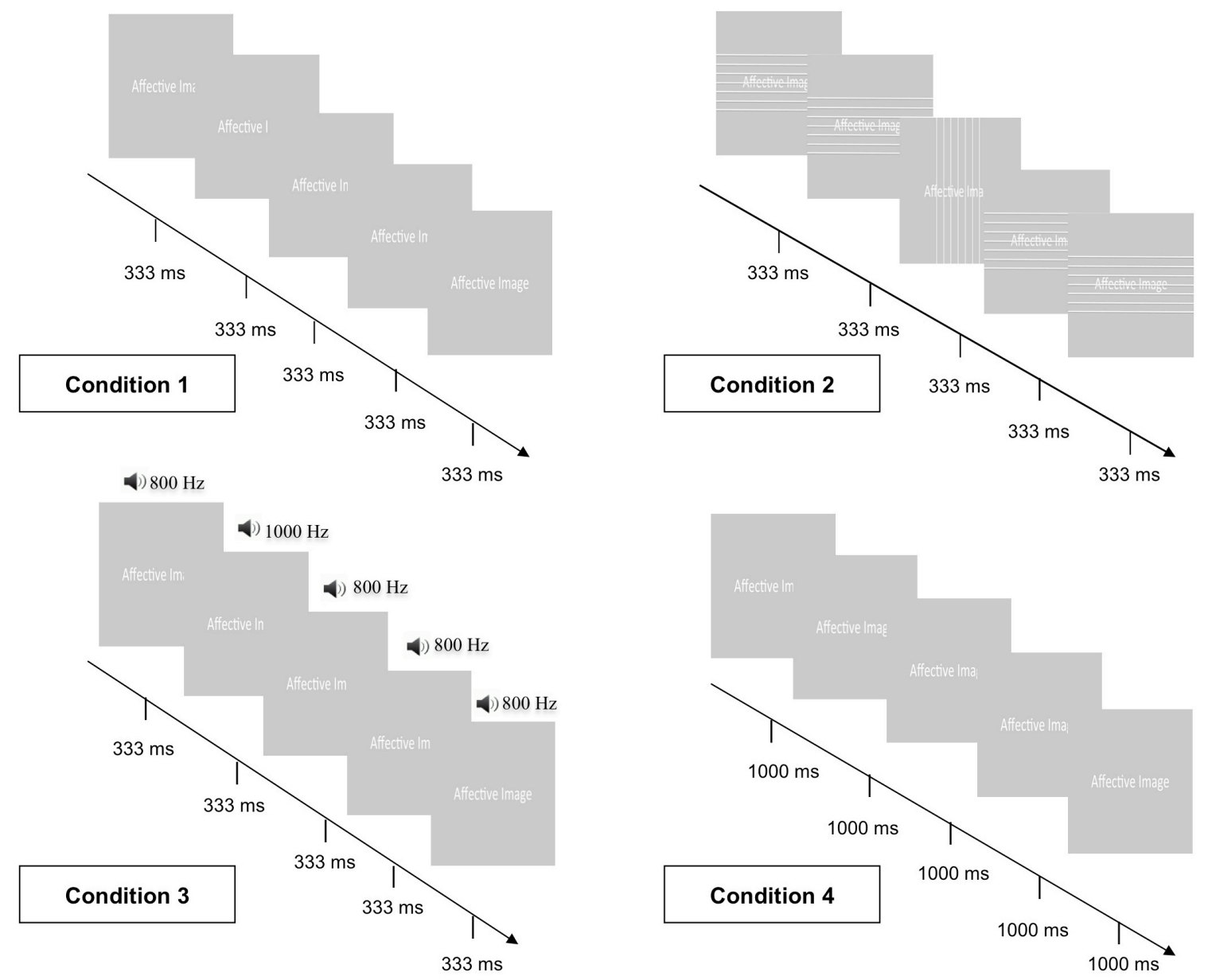

Fig. 1. Four conditions probing the EPN and LPP of emotional attention and the influence of cognitive distractions were performed. In condition 1 , the patient viewed IAPS images changed at $333 \mathrm{~ms}$ intervals to capture EPN. In condition 2, each image was overlaid by horizontal or vertical lines, and in condition 3, each image was accompanied by two different tones ( 800 or $1000 \mathrm{~Hz}$ ). In condition 4, images changed at longer intervals of $1000 \mathrm{~ms}$ to capture LPP.

vertex-referenced data set. For comparing ERP results between patients and controls, responses of each trial in each study task were calculated for all subjects in a first step to calculating a mean time range. After this, different ERP responses were calculated for each group (patients and control subjects) to distinguish possible group differences. The early selective processing for affective relative to neutral pictures was investigated in bilateral clusters over temporo-occipital areas [34]. According to each task and mean ERP responses of all recorded EEGs, we first calculated an overall grand average of all subjects and conditions to determine the exciting time ranges in which ERP responses were pronounced in each study run. In that way, the time to compare results of the early processing of affective stimuli focusing on the early posterior negativity (EPN) during implicit and during cognitive tasks should be limited to around 200 and $320 \mathrm{~ms}$ after picture onset. To extract the EPN from the EEG signal, separate average waveforms for pictures of low and high emotional arousal were calculated for each sensor and experimental condition.
The late selective processing appearing as enlarged late positive potential (LPP) amplitudes for affective relative to neutral pictures was investigated in bilateral clusters over the centro-parietal sensor area [35]. The LPP was also calculated after determining a mean time range of the grand average of all subjects, thus within a median time interval from 492 to $872 \mathrm{~ms}$ after picture onset.

Visual inspection determined that P300 waveforms were observed for the visual and the auditory attention task, differentiating an augmented P300 for target stimuli. P300 amplitude was calculated separately across separate average waveforms for each sensor of left and right centro-parietal clusters and experimental condition (non-target and target stimuli) for the visual and auditory attention task [34]. The P300 for visual stimuli was investigated in a time interval from $476 \mathrm{~ms}$ to $644 \mathrm{~ms}$, and for auditory stimuli in a time interval from 284 $\mathrm{ms}$ to $480 \mathrm{~ms}$.

\subsection{Magnetic resonance imaging}

We performed cranial magnetic resonance imaging (MRI) using a 1.0 Tesla scanner (Siemens, Erlangen) in each patient. 
T1-weighted and dual-echo T2-weighted sequences, proton MR spectra and FLAIR sequences were recorded. The image matrix size was $256 \mathrm{~m}^{2}$, the slice thickness of each axial scan was $3 \mathrm{~mm}$. Cerebral lesion volumes were calculated by the obtained sequences semi-quantitatively using Image $\mathrm{J}$ (version 1.51, LOCI, University of Wisconsin, Madison, Wisconsin, USA), which represents a highly reproducible thresholding technique [45]. Lesions were segmented on computer displayed slices by delineating the regions of interests (ROI), and lesion volume was calculated by multiplying the total ROI area with the slice thickness. Each total lesion load (total lesion volume; TLV) was calculated, whereby a separate frontal lesion load (frontal lesion volume; FLV) was considered if appropriate. According to possible impacts of the amount of TLV of MRI lesions on neuropsychologic and - physiologic data, differentiation of patients with low lesion load (low lesion volume; LLV) and high lesion load (high lesion volume; $\mathrm{HLV}$ ) with a dividing line at $3 \mathrm{~cm}$ was considered [46].

\subsection{Data analysis}

SPSS Version 16.0 (IBM Corp., Chicago, IL, USA) packaging was used for statistical analysis. Neuropsychological test results were compared by $T$-Test for independent samples and univariate analysis of variance (ANOVA), and ANOVA compared ERP data of the EEG recordings. Statistical significance was supposed for results with a level of 5\% ( $p=$ $0.05)$, and a level of $1 \%(p=0.001)$ was considered highly significant. Levene's test was applied for proving the basis of variance homogeneity. A calculation of Eta square $\left(\eta^{2}\right)$ was applied to significant statistical variables of emotion domains (ERP, TAB) only in MS patients to assess the effect size. Testing possible correlative effects of different parameters, we applied Spearman's calculation of correlations coefficients. Improving the transparency and interpretation, TAP results were displayed in two ways: (1) comparison of MS and control group, and (2) comparison of norm data of test battery ( $T$-values).

\section{Results}

\subsection{Clinical data}

All examined patients (four males and seven females) suffered from a primary relapsing-remitting course of Multiple Sclerosis (RRMS). Three out of these patients also gained the criteria of a secondary chronic progressive course of illness. The mean age of these patients was 37.4 years, and of the eleven healthy subjects was 36.5 years (see Table 1 for details). The latencies of the P100 of Visual Evoked Potentials (VEP) were $128.1 \mathrm{~ms}(+10.8)$ of the left and $126.3 \mathrm{~ms}(+10.3)$ of the right occipital derivation $(+8.5)$. The mean duration time of illness course, the mean EDSS, and the mean scores of the MSFC subitems are depicted in Table 1.

\subsection{Neuropsychological test battery}

Studying the affective discrimination abilities by the TAB, patients with MS revealed significant deficiencies for discrimination (subtest $2 ; \mathrm{t}(12)=-3.74 ; p<0.01 ; \eta^{2} 0.76$ ) and
Table 1. Overview of subjects basic data.

\begin{tabular}{lcc}
\hline & MS & Control \\
\hline Subjects (n) & 11 & 11 \\
Mean age & $37.36(+10.95)$ & $36.5(+8.5)$ \\
Sex & F: 7, M: 4 & F: 7, M: 4 \\
Mean duration of illness (years) & $8.5( \pm 4.3)$ & \\
EDSS & $2.7(+1.6)$ & \\
WD & $5.7(+3.4)$ & \\
NHPT & R: $19.1(+3.7)$ & \\
PASAT & L: $19.3(+4.0)$ & \\
\hline
\end{tabular}

Overview of demographic and clinical data of participants. Abbreviations: F, female; M, male; EDSS, Expanded Disability Status Scale; WD, walking distance; NHPT, nine-hole-peg-test (R, right side; L, left side); PASAT, Paced Auditory Serial Attention Test.

matching of facial affect expressions (subtest $5 ; \mathrm{t}(20)=-2.35$; $\left.p<0.05 ; \eta^{2} 0.87\right)$. Regarding the remaining subtests of facial expression of emotions (subtests 1, 3 and 4), MS patients also yielded lower scores than control subjects, but these were beyond a statistical significance (see Fig. 2).

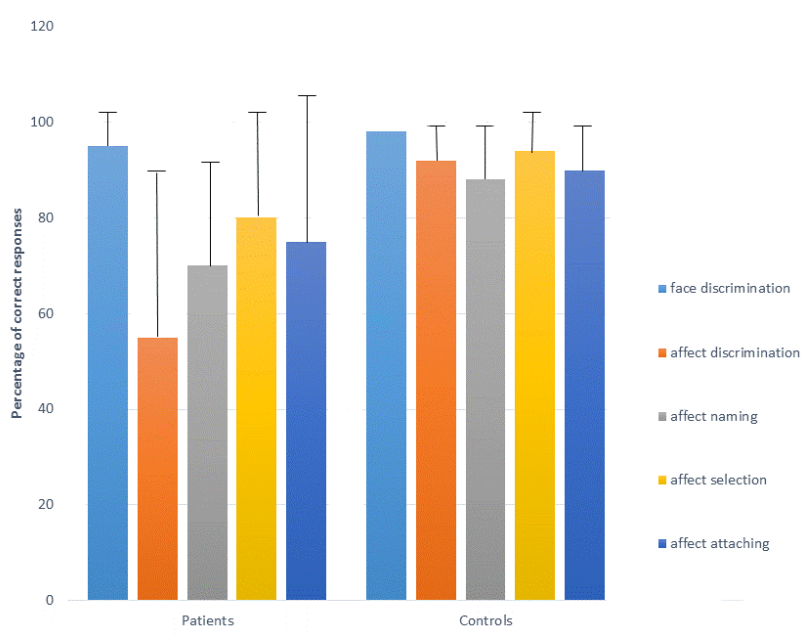

Fig. 2. Results of the Florida Affect Battery. The scores (in the percentage of valid responses) of subtest 1 up to 5 are displayed for patients at the left and controls at the right half.

Analyzing the cognitive abilities of attention, executive and memory functioning, MS patients showed impaired performance in different degrees (for a detailed overview of each result, see Table 2). In concern to attentional domains, the applied subitems of TAP revealed significant impairments in MS patients for tonic and phasic alertness with a significant main effect for the group of tonic alertness $(\mathrm{F}(1,21)=14.04 ; p$ $<0.01$ ), and also for the divided attention (significant: deficiencies for missings for general and for tones). Focusing the efforts of covered attention shifting, significant main effects were obtained for the factors condition (valid/invalid; $F(1)$ $=9.05, p<0.01)$ and group $(\mathrm{F}(1,21)=29.4, p<0.01)$, depending on reaction time. Depending on reaction time, the 
Jeton test as representing the classical Stroop effect as part of executive functioning revealed a significant main effect on factor condition (interference/non-interference: $\mathrm{F}(1,21)$ $=46.9 ; p<0.01)$ and factor group $(\mathrm{F}(1,21)=24.6 ; p<0.01)$. As a further investigation of executive functions, the CKV as the computerized version of the Wisconsin Card Sorting Test yielded significant scores for perseverations and concept perseverations (see Table 2). Also, patients exceeded the border value of false attachments by $2 \%$, whereas control subjects scored normal with $14 \%$. Finally, the memory capacities, tested by the WMS-R, exhibited significant group differences in all four memory indices (see Table 2).

\subsection{Event-related potentials}

Probing the event-relating potentials of the early capture of arousing affective contents as provided by cues of the IAPS, patients as well as controls elicited in all three conditions (1-3) an augmented early posterior negativity (EPN) over temporo-occipital regions of derivation as the neurophysiological response to pictures of high emotional arousal compared to pictures of low arousal, each displayed for $333 \mathrm{~ms}$ (condition 1).

Viewing pictures just as the primary task and focusing the attention on visual stimuli representing the secondary cognitive task (condition 2), MS patients elicited a pronounced negativity shift to highly arousing pleasant and unpleasant pictures in contrast to pictures of low arousal around temporo-occipital sites, which was beyond a clear statistical significance $(\mathrm{F}(2,54)=2.808 ; p<0.068)$. In contrast, control subjects elicited a significant pronounced negativity shift to highly arousing pleasant and unpleasant pictures at this trial $(\mathrm{F}(2,54)=7.002 ; p<0.01)$. The same ERP pattern in MS and healthy subjects remained if calculating the EPN only for emotional cues while only focusing the attention on target lines. This difference of ERP power remained significant when analyzing the between-group effect of the $\operatorname{ERP}(\mathrm{F}(2,54)$ $=7.306 ; p<0.027 ; \eta^{2} 0.76$ ). Interestingly, viewing images of negative emotion valence during competitive overlayed lines as a secondary cognitive task was negatively correlated to the sum of successively counted events of vertical lines as a target condition in MS patients $(-0.859, p<0.01)$ (see Fig. 3).

Viewing pictures simply as the primary task and focusing the attention on auditory stimuli representing the secondary cognitive task (condition 3), MS patients as well as healthy subjects elicited a significant pronounced negativity shift to highly arousing pleasant and unpleasant pictures in contrast to pictures of low arousal around temporo-occipital sites $(\mathrm{F}(2,60)=11.905 ; p<0.01 ; \mathrm{F}(2,60)=5.047 ; p<0.01)$, whereby a between-group comparison showed a stronger EPN elicitation among healthy subjects $(\mathrm{F}(2,60)=10.829 ; p<$ 0.01 ) (see Fig. 4). However, the within-group effect remains significant if considering only those EPN while focusing on the auditory target stimuli $(\mathrm{F}(2,60)=9.153 ; p<0.01 ; \mathrm{F}(2,60)$ = 9.599; $p<0.01$ ).

Analyzing the LPP during the presentation of pictures of the IAPS within a time frame of $1000 \mathrm{~ms}$ (condition 4), MS patients showed no statistically significant augmentation of the LPP over centroparietal regions to pictures of the IAPS with a highly arousing pleasant or unpleasant content in comparison to pictures with a low arousing content for the time range of 492 up to $872 \mathrm{~ms}(\mathrm{~F}(1,21)=1.503 ; p=0.231)$, as it was for control subjects $(\mathrm{F}(1,21)=10.228 ; p<0.01)$. Analyzing the LPP at a between-group comparison, this finding remained significant for healthy subjects $(\mathrm{F}(1,21)=10.080 ; p$ $<0.01$ ) (see Fig. 5).

Considering the P300 as an index of successive cognitive discrimination for the synchronously displayed non-emotion tasks, patients $(\mathrm{F}(1,21)=12.876 ; p<0.01)$ as well as control subjects $(\mathrm{F}(1,21)=8.317 ; p<0.01)$ elicited a more robust positive ERP response around $300 \mathrm{~ms}$ to optically overlayed black lines of vertical orientation, as instructed as a target cue (Fig. 2). However, the visual P300 in MS patients has negatively correlated to several modules of the neuropsychologically probed attention domains, in particular the right hemisphere to alertness (trials with a warning tone, valid reactions: $-0.725, p<0.05$; trials with a warning tone, lapses: $-0.725, p<0.05)$. ERP response to auditory target stimuli as displayed as a tone of $1000 \mathrm{~Hz}$ demarked a weak P300 for the target tones in patients $(\mathrm{F}(1,21)=2.126 ; p<0.071)$ and an augmented P300 in control subjects $(\mathrm{F}(1,21)=9.406 ; p$ $<0.01$ ). In comparison to visual P300, the auditory P300 showed some exciting correlations to clinical scores of alertness (trials with a warning tone, valid reactions, 674, $p<0.05$; trials with a warning tone, lapses: $682, p<0.05$; trials with lapses of attention: $-0.703, p<0.05$; total mean of reaction time to incompatibility: $-0.723, p<0.05)$. Behavioral data would show quite adequate attention of auditory target stimuli if a false rate of up to $20 \%$ of missing targets were accepted.

\subsection{Magnetic resonance imaging}

Processing the MRI slices of ten patients with MS (one missing data set because of personal time constraints), six patients had a total lesion load (total lesion volume; TLV) below $3 \mathrm{~cm}^{3}$ (low lesion volume; LLV), and four patients showed a total lesion load greater $3 \mathrm{~cm}^{3}$ (high lesion volume; HLV). A distinct comparison in our sample did not yield any pronounced lesion load of the frontal cortex in comparison to the total lesion load, so further correlation analyses between MRI lesion load and neuropsychological as well as ERP evaluation were only employed for the total lesion load of the cerebrum (TLV) and the MRI-split subgroups with a lesion load more significant or smaller than $3 \mathrm{~cm}^{3}$ (HLV and LLV).

Calculating correlations of MRI lesion loads for clinical scores, a positive correlation was given to the EDSS and the total lesion load (TLV) $(0.70 ; p<0.05)$. Scores of the MSFC were without a significant correlation to the total lesion load (TLV) and the MRI-split subgroups of a low or high lesion load (LLV and HLV). Correlating the MRI lesion loads to neuropsychologic data of our sample, a negative correlation between the total MRI lesion load (TLV) and the valid reactions of the overall test within the TAP $(-0.65 ; p<0.05)$ was detected. On the other hand, positive correlations have been 
Table 2. Overview of the neuropsychological results.

\begin{tabular}{|c|c|c|c|}
\hline Test item & MS subjects & Control subjects & Statistic \\
\hline \multicolumn{4}{|l|}{-Tübinger Affekt Batterie (TAB) } \\
\hline Subtest 1: face discrimination & $98 \%$ & $100 \%$ & \\
\hline Subtest 2: affect discrimination & $83 \%$ & $97 \%$ & $t(12)=-3.74 ; p<0.01$ \\
\hline Subtest 3: affect naming & $88 \%$ & $95 \%$ & \\
\hline Subtest 4: affect selecting & $93 \%$ & $98 \%$ & \\
\hline Subtest 5: affect matching & $89 \%$ & $96 \%$ & $t(20)=-2.35 ; p<0.05$ \\
\hline \multicolumn{4}{|l|}{ - Test of Attentional Performance (TAP) } \\
\hline Alertness: trials without warning tone-median of reaction time & $327 \mathrm{~ms}$ & $242 \mathrm{~ms}$ & $t(11)=2.63 ; p<0.05$ \\
\hline Alertness: trials with warning tone-median of reaction time & $345 \mathrm{~ms}$ & $230 \mathrm{~ms}$ & $t(10)=2.53 ; p<0.05$ \\
\hline Alertness: trials without warning tone-median of reaction time, $t$-value & 34.8 & 46.45 & \\
\hline Alertness: trials with warning tone-median of reaction time, $t$-value & 33.4 & 46.27 & \\
\hline D3: general test-median of reaction time & $735 \mathrm{~ms}$ & $676 \mathrm{~ms}$ & \\
\hline D3: square test-median of reaction time & $852 \mathrm{~ms}$ & $791 \mathrm{~ms}$ & \\
\hline D3: tone test-median of reaction time & $599 \mathrm{~ms}$ & $574 \mathrm{~ms}$ & \\
\hline D3: general test-missings (median) & 2.6 & 0.7 & $t(13)=2.99 ; p<0.01$ \\
\hline D3: square test-missings (median) & 0.9 & 0.5 & \\
\hline D3: tone test-missings (median) & 1.7 & 0.3 & $t(11)=2.24 ; p<0.05$ \\
\hline D3: general test-missings, $t$-value & 41 & 50 & \\
\hline D3: square test-missings, $t$-value & 49 & 53 & \\
\hline D3: tone test-missings, $t$-value & 38 & 46 & \\
\hline Incompability: general test-false reactions & 2 & 3.82 & $t(20)=-2.14 ; p<0.05$ \\
\hline Incompability: general test-false reactions, $t$-value & 54.82 & 48.64 & \\
\hline \multicolumn{4}{|l|}{-Covert Attention Shift } \\
\hline PO1: warning stimulus left/target left-median of reaction time (valid) & $366 \mathrm{~ms}$ & $280 \mathrm{~ms}$ & $t(20)=2.719 ; p<0.05$ \\
\hline PO1: warning stimulus left/target right-median of reaction time (invalid) & $442 \mathrm{~ms}$ & $322 \mathrm{~ms}$ & $t(20)=2.792 ; p<0.05$ \\
\hline PO1: warning stimulus right/target left-median of reaction time (invalid) & $405 \mathrm{~ms}$ & $312 \mathrm{~ms}$ & $t(20)=2.656 ; p<0.05$ \\
\hline PO1: warning stimulus right/target right-median of reaction time (valid) & $353 \mathrm{~ms}$ & $272 \mathrm{~ms}$ & $t(20)=2.554 ; p<0.05$ \\
\hline PO1: warning stimulus left/target left-median of reaction time (valid), $t$-value & 39 & 51 & \\
\hline PO1: warning stimulus left/target right-median of reaction time (invalid), $t$-value & 39 & 50 & \\
\hline PO1: warning stimulus right/target left-median of reaction time (invalid), $t$-value & 40 & 52 & \\
\hline PO1: warning stimulus right/target right-median of reaction time (valid), $t$-value & 41 & 50 & \\
\hline \multicolumn{4}{|l|}{ •Stroop Test } \\
\hline version 1 (first trial) & $907 \mathrm{~ms}$ & $709 \mathrm{~ms}$ & \\
\hline version 1 (second trial) & $735 \mathrm{~ms}$ & $587 \mathrm{~ms}$ & \\
\hline version 2 (third trial) & $813 \mathrm{~ms}$ & $670 \mathrm{~ms}$ & \\
\hline version 2 (fourth trial) & $809 \mathrm{~ms}$ & $644 \mathrm{~ms}$ & \\
\hline verbal interference (fifth trial) & $1004 \mathrm{~ms}$ & $850 \mathrm{~ms}$ & \\
\hline colour interference (sixth trial) & $1076 \mathrm{~ms}$ & $905 \mathrm{~ms}$ & \\
\hline verbal interference-general failure & 2.2 & 1.7 & \\
\hline verbal interference-interference failure & 1.8 & 1.5 & \\
\hline colour interference-general failure & 1 & 1.4 & \\
\hline colour interference-interference failure & 0.8 & 0.9 & \\
\hline \multicolumn{4}{|l|}{-Computer-based Card Sorting Test (CKV) } \\
\hline wrong matching & $16 \%$ & $14 \%$ & \\
\hline perseveration score & $17 \%$ & $6 \%$ & \\
\hline concept & 6 & 6 & \\
\hline concept acquisition & 6.7 & 6.6 & \\
\hline concept lost & 0.7 & 0.6 & \\
\hline concept perseverations & 0.4 & 0 & \\
\hline \multicolumn{4}{|l|}{-Wechsler Memory Scale-revised (WMS-R) } \\
\hline verbal & 93 & 108 & $t(17)=-3.13 ; p<0.01$ \\
\hline visual & 101 & 115 & $t(20)=-4.67 ; p<0.01$ \\
\hline general & 94 & 113 & $t(20)=-4.87 ; p<0.01$ \\
\hline attention and concentration & 93 & 108 & $t(20)=-3.29, p<0.01$ \\
\hline
\end{tabular}

Detailed results of the neuropsychological test battery. If a comparison between both groups were statistically significant, the pertinent score data are inserted. For more details, please see the explanations in the result section of the article. 


\section{Early Posterior Negativity (EPN): Secondary visual task}
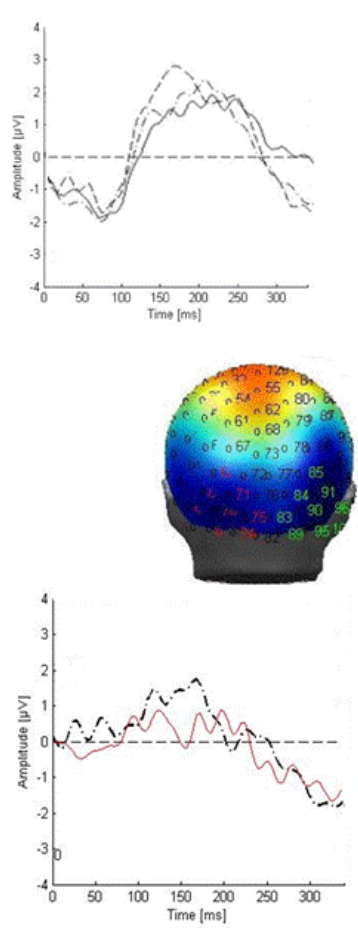
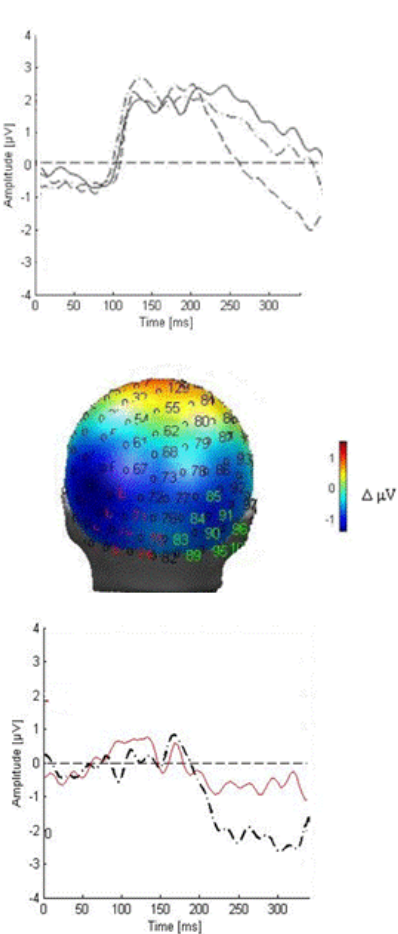

P300
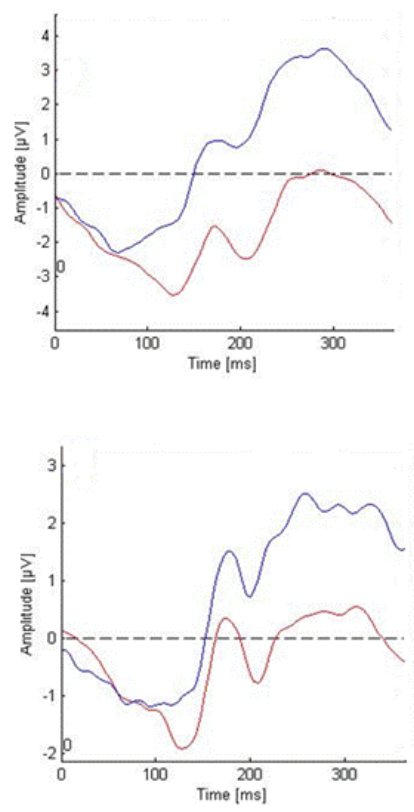

Fig. 3. Grand average of the Early Posterior Negativity (EPN) of condition 2 for competing for visual attention to affective and non-affective cues (IAPS viewing as the primary implicit task, and focusing horizontal and vertical lines as the explicit secondary task). At the left row, EPN in patients for all affective (dashed line: negative valence; dotted line: positive valence) and neutral (solid line) cues are displayed at the upper, its corresponding power map at the middle level. The EPN for affective (dashed line) and neutral (solid line) valence is displayed lower. In the same order, results of EPN for controls are displayed in the middle row. At the right row, corresponding P300 to target (blue line) and non-target (red line) cues of presented images are displayed. Time range of EPN: 200-320 ms (baseline: $-42 \mathrm{~ms}$ ).

found between the TLV and the rate of false reactions (0.69; $p<0.05)$ and missing reactions $(0.645 ; p<0.05)$ in TAP. Significant correlations between the CKV and the MRI lesion load subgroups were found for conceptual registration ( -0.68 ; $p<0.05)$ and conceptual loss $(-0.68 ; p<0.05)$, and for trends, the item all scores $(0.58 ; p=0.079)$, correct responses $(-0.60$; $p=0.068)$ and false responses $(0.60 ; p=0.068)$, perseveration $(0.57 ; p=0.085)$, and conceptual perseveration $(0.58 ; p=0.077)$, Furthermore, there was a negative correlation between the MRI lesion load subgroups and attention and concentration ($0.64 ; p<0.05)$, furthermore a positive between visual performance $(0.68 ; p<0.05)$, as tested by the Wechsler Memory Scale (WMSR). Finally, the quality of facial affect matching was negatively correlated to TLV of MRI $(-0.709 ; p<0.05)$.

Concerning correlative relations between ERP and MRI data, the auditory P300 showed a significant negative correlation to the TLV of MRI lesion in MS patients, i.e., for the amplitude of the P300 of the left centroparietal areas to the total lesion load $(-0.67 ; p<0.05)$, and the P300 of both hemispheres to the MRI-split subgroups of patients with HLV (left side: $-0.64 ; p<0.05$; right side: -0.56 ; $p=0.092$ ). Statistically relevant correlative effects of the early posterior nega- tivity and the late positive potentials to MRI lesion loads and clinical and neuropsychologic data were not found.

\section{Discussion}

We investigated eleven patients of multiple sclerosis (MS) with a predominately primary relapsing-remitting illness course (RRMS) (three already advanced to a secondary illness course) with clinically moderate functional disability and eleven healthy controls on specific affective capacities to emotion attention and empathy, but additionally also cognitive functions, by robust neurophysiological and additional neuropsychological tools. Principal electrophysiological findings of disturbed emotion modulation as displayed by indicative ERP paradigms were impairments in perceiving affective cues on early stages were apparent in conditions of concurring cognitive tasks, whereby impairments in processing affective cues at late stages were already detectable without comprising concurrent explicit cognitive processing efforts. Therefore, our ERP findings suggest disturbed bottom-up and top-down processing lines of implicit encoding of affective cues in MS patients of our study due to reduced available neural resources of attentional domains to 


\section{Early Posterior Negativity (EPN): Secondary auditory task}
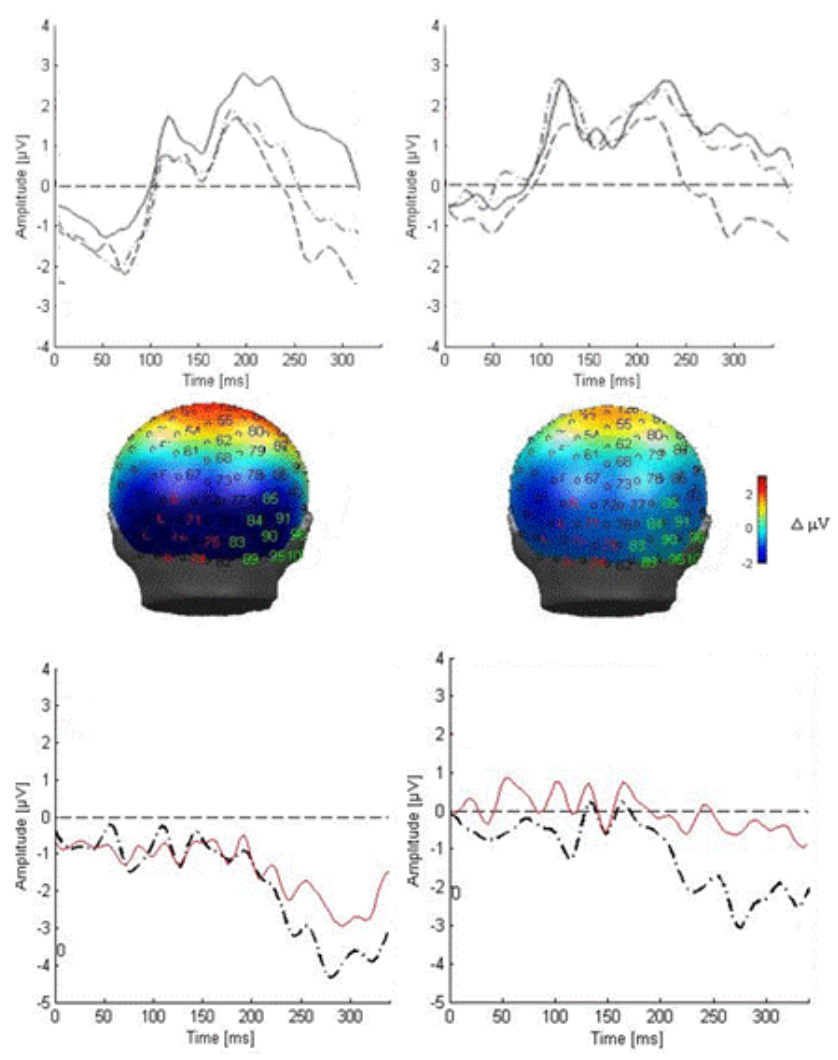

P300
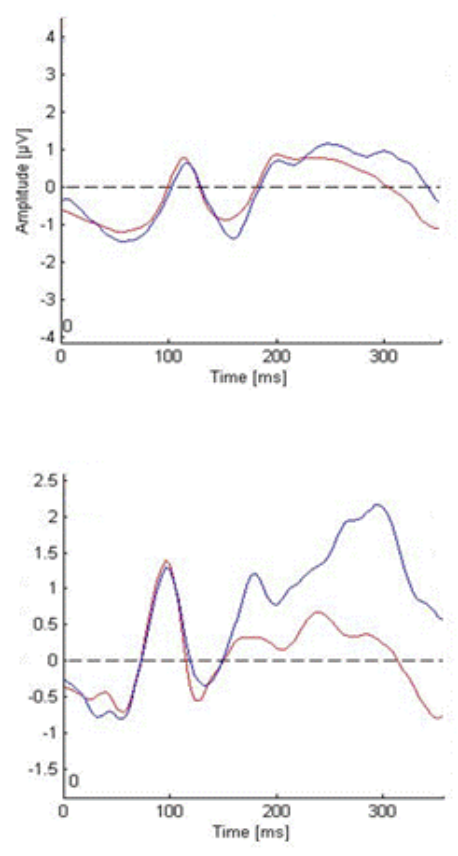

Fig. 4. The Early Posterior Negativity (EPN) of condition 3 for competing for attention to viewing affective and non-affective cues as the primary implicit task and listening to differing tones $(800 \mathrm{vs.} 1000 \mathrm{~Hz})$ as the explicit secondary task. Similar to Fig. 3, EPN in patients for all affective (dashed line: negative valence; dotted line: positive valence) and neutral (solid line) cues are displayed at the upper level of the left row, and its corresponding power map at its middle level. The EPN for affective (dashed line) and neutral (solid line) valence is displayed at the lower-left level. EPN for controls is displayed in the middle row. At the right row, corresponding P300 to target (1000 Hz, blue line) and non-target ( $800 \mathrm{~Hz}$, red line) cues are displayed. Time range of EPN: $200-320 \mathrm{~ms}$ (baseline: $-42 \mathrm{~ms}$ ).

emotional and cognitive cues at different but sequential processing stages. The recorded visual and auditory P300 suggested a preserved modulation in patients with a low MRI lesion volume and a disturbed modulation in patients with a high MRI lesion load. These electrophysiological findings in our sample of MS patients were accompanied by clinical impairments in emotion recognition in terms of discrimination and matching of emotion face expressions as proved by the Tübinger Affekt Batterie (TAB). Concerning cognitive domains, neuropsychological findings in MS patients of our study indicated cognitive impairments and several domains of memory, attention and executive functions, accompanied by an increased reaction time in nearly all neuropsychological test items. However, the neuropsychological findings of the cognitive domains did not correlate to the electrophysiological impairments as displayed by the affective ERP paradigms of the early posterior negativity (EPN), the late positive potential (LPP), and the P300 to non-emotional cues.
Our ERP findings, delineating aberrant neurophysiological processing of affective cues with salient contents of differing valence and arousal in subjects suffering from a predominately subcortical lesioning, are quite interesting in terms of the repeatedly reported ERP modulation for emotions of different valence and arousal apparent over temporooccipital and parietal areas in early and late stages of encoding $[31,34]$. The prominent feature is early negativity (EPN) over temporo-occipital regions within a time range between 150 and $300 \mathrm{~ms}$ after picture onset, which is accompanied by an augmentation for contents of evolutionary importance (erotics, mutilations or threats), reflecting the intensity of affective engagement of motivational systems such as approachment or avoidance within an initial transitory processing phase of selected elaboration processing of emotional stimuli [47]. Source analysis suggests an activation of the extended visual system, indexing an early facilitated sensory processing of affective cues [33]. Encoding af- 


\section{Late Positive Potential (LPP)}
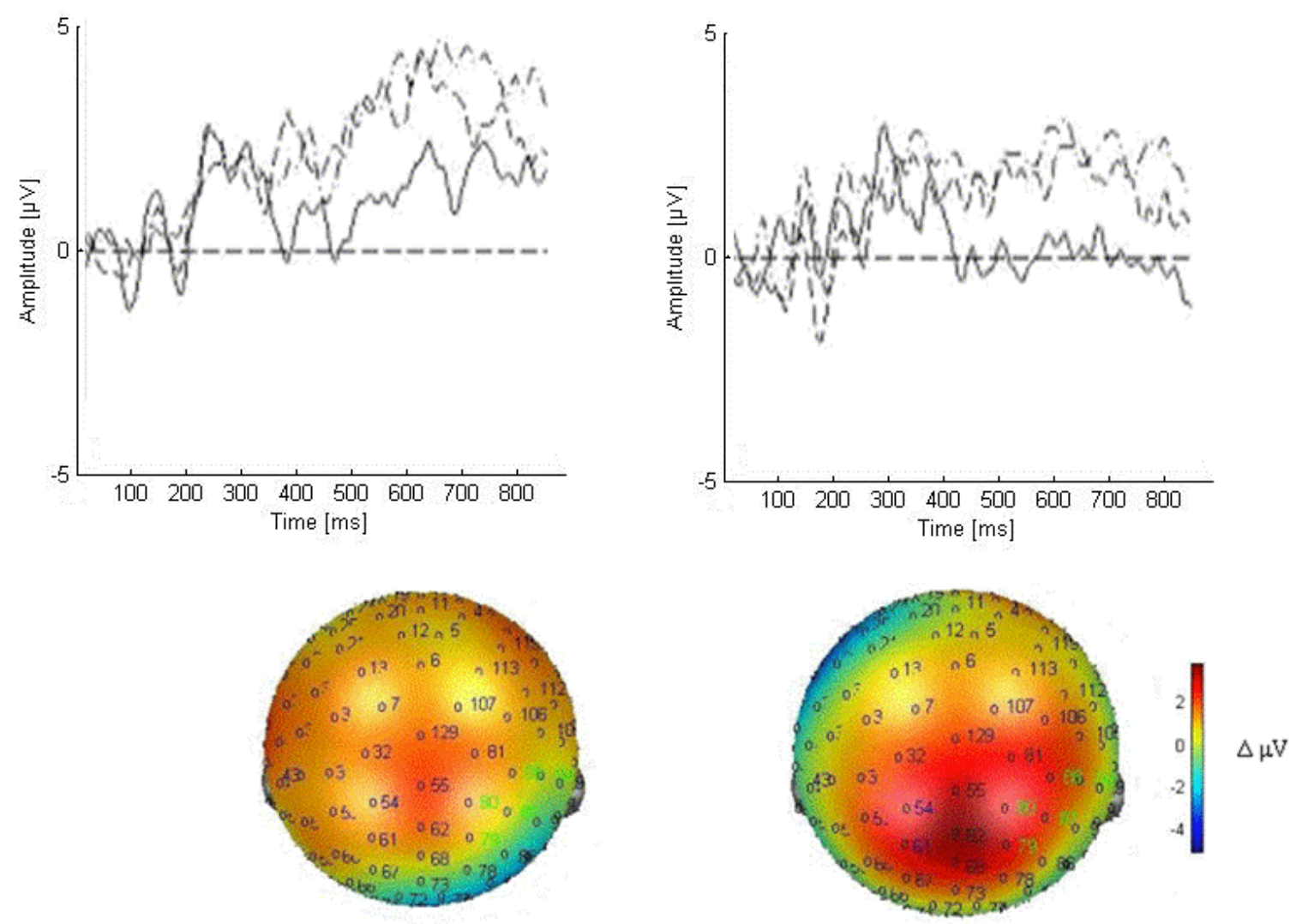

Fig. 5. Grand-averaged Late Positive Potential (LPP) of condition 4 for viewing pictures of the IAPS with different emotional valence (pleasant: dashed line: unpleasant: dashed-dotted line: neutral: solid line) as an implicit task. ERP and its corresponding power map of patients are depicted at the left row, and ERP and power map of controls are depicted at the right row. Time range of LPP: 492 to $872 \mathrm{~ms}$.

fective cues at these early stages reflects an automatic routine of capturing emotional stimuli independent of top-down control [34]. Indeed, intracranial recordings at inferior temporal and fusiform gyrus described a robust object-selectivity during initial processing (around $200 \mathrm{~ms}$ ), despite a second object within the image [48]. Onward processing of visually presented affective cues with higher emotional arousal is associated with enlarged late positive potentials (LPP) relative to low arousing ones over parietal areas, indicating an intrinsically higher relevance of affective cues relative to neutral objects' high-order stages stimuli processing [35]. According to these well-evaluated findings of neurophysiologically detectable features of implicit affective processing, our sample of MS patients showed an impaired implicit emotional discrimination to presented visual affective cues of evolutionary relevance at the early stage of stimuli encoding in concurrently forced attention to non-affective cues, delineating the limitations of cue processing at bottom-up stages if the attentional load is subject of a critical threshold of available neural resources mainly demanded selected attention.
The failure of augmented LPP in our patient sample suggests a psychophysiological feature of disturbed implicit processing of affective cues at onwarding high-order stages, indicating a compromised allocation of processing resources in a capacity-limited processing stage associated with stimulus representation working memory $[35,49,50]$. Similarly, the reduced EPN to emotion cues in cases of forced selective attention to non-emotion objects indicates interference effects of competition for shared processing of those concurrent implicit emotional and explicitly cognitive tasks in the visual stream, unmasking a limited availability of processing resources for high-level constraints and thus unmasking the obligatory discriminative capturing of emotional significance in our sample of MS patients [51,52]. The preferential processing of high-priority stimuli in the environment is an essential function of selective attention. Still, an interitem competition among neural maps in the ventral stream of cue attention representing foreground task-stimuli and background emotion stimuli, as applied in our paradigm, obviously differed in favor of top-down attention processes for 
focusing visual attention on task-relevant central stimuli so that emotionally relevant stimuli could no longer elicit the ERP signature of preferential emotion processing in our patients $[47,53]$. Nevertheless, the finding of an augmented EPN to affective cues with a less constraining straightforward task suggests some preserved resources of visual attention to affective cues of evolutionary relevance along bottomup pathways, similar to the observation of long preserved resources of hierarchical lower attention, such as attention span in patients with MS within the early phase of illness course $[12,35]$.

Concerning our neuropsychological findings of impairments in attention functions, these observations might be of interest in the discrepancy of the lesion-depending alterations of the P300 in our patient sample. ERP such as the P200 and the P300 is of particular interest for studying specific neurophysiological patterns of cognitive impairment in MS. A recent study of MS patients in a study by WaliszewskaProsol et al. [54] showed higher P200 amplitudes, suggesting increased cortical and subcortical activity as a compensatory mobilization of more extensive neural networks to ensure better stimulus analysis. Accordingly, Senkowski and Herrmann [55] found a more substantial P200 amplitude for tasks with a higher difficulty level of a visual discrimination task. On the other hand, as a frequently analyzed ERP component in cognitive neuroscience, P300 is often associated with increased latency and/or decreased amplitude of this ERP, even suggesting a prognostic indicator for the progression of cognitive impairment in MS [56]. Honig et al. [57], and Piras et al. [58], argued that the consideration of structural issues in cognitive decline is relevant. They found a close correlation between changes in visual and auditory P300 and the number of plaques (lesion load) in MS [57, 58]. Thus, finding reduced P300 amplitude in auditory target stimuli at left parietal sites in MS patients with higher lesion load on MRI, as found in our study sample, is more consistent with the cognitive impairment neuropsychologically observed in the patients. This observation reflects the limitations or susceptibility of the functional complexity of the cerebral system in directing attention efforts to working memory, as seen, for example, in a study of working memory with the Sternberg memory scanning task in MS [59]. There, patients with lower scores in neuropsychological tests of working memory also showed a reduced positive shift in their reactions to memory probes. The reduced positivity shift was more strongly accentuated for auditory stimuli and related more to the probably higher demand on phonological working memory. Correspondingly, other clinical findings often pointed to signs of a disturbed phonological loop in patients with MS. The findings in our study seem to be associated with a reduced capacity of verbal working memory [60].

Our neuropsychological results are in line with previous findings of neurocognitive disturbances in patients with MS $[1,13,61]$. Patients in our sample showed impairments of different neuropsychological capacities, particularly within attentional and memory and executive domains. Furthermore, patients were associated with impairments of processing resources of recognizing emotions in facial expressions, which is in line with the previous findings [10, 14, 62, 63]. Recent works indicated that these deficits also occurred in those with intact facial recognition, suggesting a rather specific affective than perceptual impairment [62-65]. These studies identified specific deficits in labeling sad, fearful, and angry facial expressions, as we found in our sample of MS patients performing the TAB. Despite the strong evidence of the neural substrates of impaired discrimination of emotional characteristics of facial expressions, in the particular prefrontal cortex (PFC) with the dorsolateral and ventromedial prefrontal cortex (DLPFC, VMPFC), the anterior cingulate and the amygdala-hippocampus-complex [66-68] disturbed transfers of responsible networks including interhemispheric transfer pathways of affective information may crucially account for impaired affect recognition and its significance in social cognition in patients with MS.

The analysis of correlations between neuropsychological and neurophysiological impairments to brain lesions in our considered cranial MRI was dominated by cognitive rather than affective impairments. This observation remains ambiguous concerning the different variables of affective behavior applied in our study sample, so there was a significant negative correlation to lesion load and emotion recognition in the TAB, but not for the ERP data. This observation of restricted correlations to MRI patterns for impaired emotion domains may be ascribed to the heterogeneity of topographic distribution [59] and properties of functional compensation [69]. However, several studies on the impact of brain lesions along topographic peculiarities like the number of brain lesions were often beyond a consistent relation to clinical issues, which have been assumed sequelae of neural reserves with strong compensatory potentials to cover the loss of network integrity. On the other hand, conventional MRI might be less sensitive to assess certain high order domains, so advanced MRI with higher resolution of affected brain tissues might be recommended in studying affective disturbances in MS, as microstructural measures in normalappearing white matter indeed provide more neuroimaging information [20, 70]. Nevertheless, the lower yield of correlative effects between MRI and neurophysiological data of emotion processing in our study may also be a matter of our small patient sample, so further investigations using similar or at least comparable ERP protocols in study samples with a greater amount of patients might be of interest in clarifying this issue.

\section{Limitations}

Our study results are subject to some restrictions which should be considered. The power of our study results might be subject to relatively small sample size, i.e., eleven patients and eleven controls, which calls for a further evaluation of our study protocol even to a representative sample size. The 
fact that three patients already met an advance to a secondary illness course in the meantime might be of less data impact if considering the moderate clinical degree of impairment as displayed by the EDSS. Nevertheless, we study a relatively homogenous sample of a particular brain disorder by applying robust clinical and neurophysiological study items, which delivers statistically feasible data, suggesting a valid subject in calculating the primarily hypothesized assumption of affective disturbances in MS. Therefore, further studies with a comparable protocol and a greater sample size might not only replicate but even differentiate some details of our findings due to a more representative statistical power. Another critical point of our study might be to concern the tremendous amount of variables in our protocol, i.e., besides the ERP trials in studying the neurophysiological appearance of affective disorders, accompanied by a broad set of neuropsychological tests and MRI data. To better understand our specific protocol, it is crucial to consider the growing use of clinical, neurophysiological and neuroradiological measurement tools in diagnosing and critical evaluation of individually afflicted functional areas in MS patients. Indeed, the optimal amount of chosed variables in characterizing such domains of affect regulation and possible cognitive influences like attentional and executive functions might challenge future research in MS.

\section{Conclusions}

The present work identified specific neurophysiological features of impairments in emotional attention and clinical features of a disorder in the discrimination of affective facial expressions, which contribute interesting information to the background and understanding of the neural mechanisms of affective disorders in MS. The functional deficits in our sample, which can be assumed to be moderate overall at an EDSS of 2.7, corresponding to the discrepancy in the maintenance of attentional performance for simple demand levels, which may already be the subject of compensatory neuronal activities the implemented networks of emotion regulation. Our approach to studying specific ERP to the neurophysiological underpinnings of impaired affective processing deems to be performed here the first time, particularly highlighting our results and their weighting, mainly topographical rather than temporal aspects of emotion processing in MS have become known. The detection of specific ERP in MS to assess the neurophysiological background and thus the degree of neuronal degeneration could therefore be of interest in the early course of the disease, but a growing topic for calculating the individual potential of disease progression in affective abilities. Noteworthy, in contrast to the well-documented cognitive impairments, affective disorders are detected comparatively even less frequently, despite their decisive influence on neuropsychiatric entities such as depressive or anxiety disorders and core aspects of social cognition such as emotion recognition in facial expression or the Theory of Mind [14, 15, 63]. Specific studies of affective impairment, as provided by sensitive clinical inventories and specific neurophysiological markers such as the ERP of early and late emotion processing stages, can be used for individual statements on the therapeutic efficacy of drug modifying therapeutics, neurorehabilitative and psychotherapeutic applications to achieve a conflict resolution in favor of a decrease in the premature consumption of neuronal reserves in cognitive and affective networks $[20,71]$.

\section{Abbreviations}

ANOVA, Analysis of Variance; CKV, Computergestütztes KartensortierVerfahren; DLPFC, dorsolateral prefrontal cortex; EDSS, Expanded Disability Severity Scale; EEG, Electroencephalography; EPN, Early Negative Potential; ERP, Event-related potentials; IAPS, International Affective Picture System; FLAIR, Fluid-attenuated inversion recovery; ICD10, International Classification of Diseases, Version 10; LPP, Late Positive Potentials; MRI, Magnetic Resonance Imaging; MS, Multiple Sclerosis; MSFC, Multiple Sclerosis Functional Composite; RRMS, relapsing-remitting course of multiple sclerosis; TAP, Test of Attentional Performance; TAB, Tübinger Affekt Batterie; VAP, Visual-evoked potentials; VMPFC, ventromedial prefrontal cortex; WMS-R, revised Wechsler Memory Test.

\section{Author contributions}

MA, SK, CK and AH designed the research study. MA and SK performed the research. NH provided help and advice on the MRI recordings. MA and SK analyzed the data. MA, SK and CK wrote the manuscript. All authors contributed to editorial changes in the manuscript. All authors read and approved the final manuscript.

\section{Ethics approval and consent to participate}

All human participants were raised and handled at the Department of Neurology and the Department of Biological Psychology, both University of Greifswald. All human experiments were carried out in accordance with the guidelines of the European Community Council Directives 86/609/EEC and approved by the Mecklenburg-Vorpommern government and the Ethical Committee of the University of Greifswald. The approvals of study participation were obtained with the informed consent of all participants. The institutional review board of the Ethical Committee of the University of Greifswald approved this study.

\section{Acknowledgment}

We are grateful for the support of Mr Alexander Dressel in cooperating by his ambulance of Multiple Sclerosis with important ideas, methodological assistance and patient selection. Furthermore, we highly thank Matthias Weymar for technical assistance. Finally, we thank two anonymous reviewers for excellent criticism of the article. 


\section{Funding}

This research was supported by grants from the Society of Neurology Mecklenburg-Vorpommern (Ada 11-05-01) for the first author. The supporters had no role in study design, data collection and analysis, decision to publish, or preparation of the manuscript.

\section{Conflict of in terest}

The authors declare no conflict of interest.

\section{References}

[1] Ryan L, Clark CM, Klonoff H, Li D, Paty D. Patterns of cognitive impairment in relapsing-remitting multiple sclerosis and their relationship to neuropathology of magnetic resonance images. Neuropsychology. 1996; 2: 176-193

[2] Aupperle RL, Beatty WW, Shelton FDNAP, Gontkovsky ST. Three screening batteries to detect cognitive impairment in multiple sclerosis. Multiple Sclerosis. 2002; 8: 382-389.

[3] Rao SM, Leo GJ, Ellington L, Nauertz T, Bernardin L, Unverzagt F. Cognitive dysfunction in multiple sclerosis.: II. Impact on employment and social functioning. Neurology. 1991; 41: 692-696.

[4] Amato MP. Cognitive Impairment in Early-Onset Multiple Sclerosis: Pattern, predictors, and impact on everyday life in a 4-year follow-up. Archives of Neurology. 1995; 52: 168.

[5] Higginson CI, Arnett PA, Voss WD. The ecological validity of clinical tests of memory and attention in multiple sclerosis. Archives of Clinical Neuropsychology. 2000; 15: 185-204.

[6] Beatty WW, Orbelo DM, Sorocco KH, Ross ED. Comprehension of affective prosody in multiple sclerosis. Multiple Sclerosis. 2003; 9: 148-153.

[7] Ruet A, Deloire M, Charré-Monn J, Hamel D, Brocher B. Cognitive impairment differ between primary progressive and relapsing-remitting MS. Neurology. 2013; 80: 1501-1508

[8] Filley CM, Heaton RK, Nelson LM, Burks JS, Franklin GM. A comparison of dementia in Alzheimer's disease and multiple sclerosis. Archives of Neurology. 1989; 46: 157-161.

[9] Rao SM, Grafman J, DiGiulio D, Mittenberg W, Bernardin L, Leo GJ, et al. Memory dysfunction in multiple sclerosis: its relation to working memory, semantic encoding, and implicit learning. Neuropsychology. 1993; 7: 364-374.

[10] Beatty WW, Goodkin DE, Monson N, Beatty PA. Cognitive disturbances in patients with relapsing remitting multiple slerosis. Archives of Neurology. 1989; 46: 1113-1119

[11] Kail R. Speed of information processing in patients with multiple sclerosis. Journal of Clinical and Experimental Neuropsychology. 1998; 20: 98-106.

[12] Paul RH, Beatty WW, Schneider R, Blanco C, Hames K. Impairments of attention in individuals with multiple sclerosis. Multiple Sclerosis. 1998; 4: 433-439.

[13] Chiaravalloti ND, DeLuca J. Cognitive impairments in multiple sclerosis. The Lancet Neurology. 2008; 7: 1139-1151

[14] Lenne B, Barthelemy R, Nandrino J, Sequeira H, Pinti A, Mecheri $\mathrm{H}$, et al. Impaired recognition of facial emotional expressions in Multiple Sclerosis. Neuropsychological Trends. 2014; 3: 67-83.

[15] Murphy R, O'Donoghue S, Counihan T, McDonald C, Calabresi PA, Ahmed MA, et al. Neuropsychiatric syndromes of multiple sclerosis. Journal of Neurology, Neurosurgery, and Psychiatry. 2017; 88: 697-708.

[16] Ruffman T, Henry JD, Livingstone V, Phillips LH. A meta-analytic review of emotion recognition and aging: implications for neuropsychological models of aging. Neuroscience and Biobehavioral Reviews. 2008; 32: 863-881.

[17] Berg D, Supprian T, Thomae J, Warmuth-Metz M, Horowski A, Zeiler B, et al. Lesion pattern in patients with multiple sclerosis and depression. Multiple Sclerosis. 2000; 6: 156-162.

[18] Bakshi R, Czarnecki D, Shaikh ZA, Priore RL, Janardhan V,
Kaliszky Z, et al. Brain MRI lesions and atrophy are related to depression in multiple sclerosis. Neuroreport. 2000; 11: 1153-1158.

[19] Dineen RA, Vilisaar J, Hlinka J, Bradshaw CM, Morgan PS, Constantinescu CS, et al. Disconnection as a mechanism for cognitive dysfunction in multiple sclerosis. Brain. 2009; 132: 239-249.

[20] Sokolov AA, Grivaz P, Bove R. Cognitive Deficits in Multiple Sclerosis: Recent Advances in Treatment and Neurorehabilitation. Current Treatment Options in Neurology. 2018; 20: 53.

[21] Nigro S, Passamonti L, Riccelli R, Toschi N, Rocca F, Valentino $\mathrm{P}$, et al. Structural 'connectomic' alterations in the limbic system of multiple sclerosis patients with major depression. Multiple Sclerosis. 2015; 21: 1003-1012.

[22] Sabatini U, Pozzilli C, Pantano P, Koudriavtseva T, Padovani A, Millefiorini E, et al. Involvement of the limbic system in multiple sclerosis with depressive disorders. Biological Psychiatry. 1996; 39: 970-975.

[23] Pujol J, Bello J, Deus J, Marty-Vilalta JL, Capdevila A. Lesions in the left arcuate fasciculatus region and depressive symptoms in multiple sclerosis. Neurology. 1997; 49: 1105-1110

[24] Tourtellotte WW, Syndulko K, Jennings P. Use of P300 and a dementia rating scale in the evaluation of cognitive dysfunction in MS. Acta Neurologica Scandinavica Supplementum. 1984; 101: 32-34.

[25] Newton MR, Barrett G, Callanan MM, Towell AD. Cognitive event-related potentials in multiple sclerosis. Brain. 1989; 112: 1637-1660.

[26] Polich J, Romine JS, Sipe JC, Aung M, Dalessio DJ. P300 in multiple sclerosis: a preliminary report. International Journal of Psychophysiology. 1992; 12: 155-163.

[27] Triantafyllou NI, Voumvourakis K, Zalonis I, Sfagos K, Mantouvalos $\mathrm{V}$, Malliara $\mathrm{S}$, et al. Cognition in relapsing-remitting multiple slerosis: A multichannel event-related potential (P300) study. Acta Neurologica Scandinavica. 1992; 85: 10-13.

[28] Van Dijk JG, Jennekens-Schinkel A, Caekebeke JF, Zwindermann $\mathrm{AH}$. Are event-related potentials in multiple slerosis indicative of cognitive impairment? Evoked and event-related potentials, psychometric testing and response speed: a controlled study. Journal of the Neurological Sciences. 1992; 109: 18-24.

[29] Gil R, Zai L, Neau JP, Jonveaux T, Agbo C, Rosolacci T, et al. Event-related auditory evoked potentials and multiple sclerosis. Electroencephalography and Clinical Neurophysiology. 1993; 88: 182-187.

[30] Leocani L, Comi G. Neurophysiological investigations in multiple sclerosis. Current Opinion in Neurology. 2000; 13: 255-261.

[31] Schupp HT, Junghöfer M, Weike AI, Hamm AO. The selective processing of briefly presented affective pictures: an ERP analysis. Psychophysiology. 2004; 41: 441-449.

[32] Lang PJ, Bradley MM, Cuthbert BN. International Affective Picture System (IAPS): Instruction manual and affective ratings. Technical report A-4. The Center for Research in Psychophysiology, University of Florida: Gaineville, FL. 1999.

[33] Junghöfer M, Bradley MM, Elbert TR, Lang PJ. Fleeting images: a new look at early emotion discrimination. Psychophysiology. 2001; 38: 175-178.

[34] Schupp HT, Junghöfer M, Weike AI, Hamm AO. Attention and emotion: an ERP analysis of facilitated emotional stimulus processing. Neuroreport. 2003; 14: 1107-1110.

[35] Schupp HT, Cuthbert BN, Bradley MM, Cacioppo JT, Ito T, Lang $\mathrm{PJ}$. Affective picture processing: the late positive potential is modulated by motivational relevance. Psychophysiology. 2000; 37: 257-261.

[36] Olofsson JK, Nordin S, Sequeira H, Polich J. Affective picture processing: an integrative review of ERP findings. Biological Psychology. 2008; 77: 247-265.

[37] Adamaszek M, Olbrich S, Kirkby KC, Woldag H, Willert C, Heinrich $\mathrm{A}$. Event-related potentials indicating impaired emotional attention in cerebellar stroke-a case study. Neuroscience Letters. 2013; 548: 206-211.

[38] McDonald WI, Compston A, Edan G, Goodkin D, Hartung HP, 
Lublin FD, et al. Recommended diagnostic criteria for multiple sclerosis: guidelines from the International Panel on the diagnosis of multiple sclerosis. Ann Neurol. 2001; 50: 121-127.

[39] Polman CH, Reingold SC, Banwell B, Clanet M, Cohen JA, Filippi M, et al. Diagnostic criteria for multiple sclerosis: 2010 Revisions to the McDonald criteria. Annals of Neurology. 2011; 69: 292302 .

[40] Cutter GR, Baier ML, Rudick RA, Cookfair DL, Fischer JS, Petkau $\mathrm{J}$, et al. Development of a multiple sclerosis functional composite as a clinical trial outcome measure. Brain. 1999; 122: 871-882.

[41] Breitenstein C, Daum I, Ackermann H, Lütgehetmann R, Müller E. Erfassung der Emotionswahrnehmung bei zentralnervösen Läsionen und Erkrankungen: Psychometrische Gütekriterien der “Tübinger Affekt Batterie”. Neurologische Rehabilitation. 1996; 2: 93-101. (In German)

[42] Zimmermann P, Fimm B. Testaufmerksamkeitsbatterie zur Aufmerksamkeitsprüfung (TAP). Psytest-Verlag: Herzogenrath. 1995;

[43] Drühe-Wienholt CM, Wienholt W. Computerisiertes Kartensortierverfahren (CKV). Hogrefe Verlag: Testzentrale. 2011.

[44] Wechsler D. Memory-Scale revised manual. I no Psychological Corporation: San Antonio. 1980;

[45] Rasband W. Image J. vl.27. National Institute of Mental Health, Bethesda, Maryland, USA. 2002. Available at: http://rsb.info.nih .gov/ij (Accessed: 11 November 2004).

[46] Sailer M, Heinze HJ, Tendolkar I, Decker U, Kreye O, Rolbicki $\mathrm{U}$, et al. Influence of cerebral lesion distribution on event-related brain potentials in multiple sclerosis. Journal of Neurology. 2001; 248: 1049-1055.

[47] Schupp HT, Schmälzle R, Flaisch T. Explicit semantic stimulus categorization interferes with implicit emotion processing. Social Cognitive and Affective Neuroscience. 2014; 9: 1738-1745.

[48] Agam Y, Liu H, Papanastassiou A, Buia C, Golby AJ, Madsen JR, et al. Robust selectivity to two-object images in human visual cortex. Current Biology. 2010; 20: 872-879.

[49] Herrmann MJ, Huter T, Müller F, Mühlberger A, Pauli P, Reif A, et al. Additive effects of serotonin transporter and tryptophan hydroxylase-2 gene variation on emotional processing. Cerebral Cortex. 2007; 17: 1160-1163.

[50] Sabatinelli D, Keil A, Frank DW, Lang PJ. Emotional perception: correspondence of early and late event-related potentials with cortical and subcortical functional MRI. Biological Psychology. 2013; 92: 513-519.

[51] Pessoa L. To what extent are emotional visual stimuli processed without attention and awareness? Current Opinion in Neurobiology. 2005; 15: 188-196.

[52] Rousselet GA, Thorpe SJ, Fabre-Thorpe M. How parallel is visual processing in the ventral pathway? Trends in Cognitive Sciences. 2004; 8: 363-370.

[53] Franconeri SI, Alvarez GA, Cavanagh P. Flexible cognitive resources: competitive content maps to attention and memory. Trends in Cognitive Sciences. 2013; 17: 134-141.

[54] Waliszewska-Prosół M, Nowakowska-Kotas M, Kotas R, Bańkowski T, Pokryszko-Dragan A, Podemski R. The relationship between event-related potentials, sress perception and personality type in patients with multiple sclerosis without cognitive impairment: a pilot study. Advances in Clinical and Experimental Medicine. 2018; 27: 787-794.

[55] Senkowski D, Herrmann CS. Effects of task difficulty on evoked gamma activity and ERPs in a visual discrimination task. Clinical Neurophysiology. 2002; 113: 1742-1753.
[56] Sundgren M, Nikulin VV, Maurex L, Wahlin Å, Piehl F, Brismar T. P300 amplitude and response speed relate to preserved cognitive function in relapsing-remitting multiple sclerosis. Clinical Neurophysiology. 2015; 126: 689-697.

[57] Honig LS, Ramsay RE, Sheremata WA. Event-related potential P300 in multiple sclerosis. Relation to magnetic resonance imaging and cognitive impairment. Archives of Neurology. 1992; 49: 44-50.

[58] Piras MR, Magnano I, Canu EDG, Paulus KS, Satta WM, Soddu A, et al. Longitudinal study of cognitive dysfunction in multiple sclerosis: neuropsychological, neuroradiological, and neurophysiological findings. Journal of Neurology, Neurosurgery, and Psychiatry. 2003; 74: 878-885.

[59] Fischer JS, Jacobs LD, Cookfair DL. Heterogeneity of cognitive dysfunction in multiple sclerosis. Clinical Neuropsychologist. 1998; 12: 286.

[60] Pelosi L, Geesken JM, Holly M, Hayward M, Blumhardt LD. Working memory impairment in multiple sclerosis. Evidence from event-related potential study of patients with clinically isolated myelopathy. Brain. 1997; 120: 2039-2058.

[61] Amato MP, Ponziani G, Siracusa G, Sorbi S. Cognitive dysfunction in early-onset multiple sclerosis: a reappraisal after 10 years. Archives of Neurology. 2001; 58: 1602-1606.

[62] Berneiser J, Wendt J, Grothe M, Kessler C, Hamm AO, Dressel A. Impaired recognition of emotional facial expression in patients with multiple sclerosis. Multiple Sclerosis and Related Disorders. 2014; 3: 482-488.

[63] Cotter J, Firth J, Enzinger C, Kontopantelis E, Yung AR, Elliott R, et al. Social cognition in multiple sclerosis. Neurology. 2016; 87: 1727-1736.

[64] Cecchetto C, Aiello M, D’Amico D, Cutuli D, Cargnelutti D, Eleopra $\mathrm{R}$, et al. Facial and bodily emotion recognition in multiple sclerosis: the role of alexithymia and other characteristics of the disease. Journal of the International Neuropsychological Society. 2014; 20: 1004-1014.

[65] Prochnow D, Donell J, Schäfer R, Jörgens S, Hartung HP, Franz M, et al. Alexithymia and impaired facial affect recognition in multiple sclerosis. Journal of Neurology. 2011; 258: 1683-1688.

[66] Hempel A, Hempel E, Schonknecht P, Stippich C, Schroder J. Processing of emotional face expressions: evaluation of healthy subjects and patients of schizophrenia by functional MRI. Nervenheilkunde. 2002; 21: 410-413.

[67] Adolphs R. Processings of Emotional and Social Information by the Human Amygdala. The New Cognitive Neurosciences III (pp. 1005-1016). 3rd edn. Gazzaniga MS (Editor-in-chief). MIT Press: Massachusetts. 2004.

[68] Prochnow D, Höing B, Kleiser R, Lindenberg R, Wittsack H-, Schäfer $R$, et al. The neural correlates of affect reading: an fMRI study on faces and gestures. Behavioural Brain Research. 2013; 237: 270-277.

[69] Staffen W, Mair A, Zauner H, Unterrainer J, Niederhofer H, Kutzelnigg A, et al. Cognitive function and fMRI in patients with multiple sclerosis: evidence for compensatory cortical activation during an attention task. Brain. 2002; 125: 1275-1282.

[70] Bonnier G, Roche A, Romascano D, Simioni S, Meskaldji D, Rotzinger D, et al. Advanced MRI unravels the nature of tissue alterations in early multiple sclerosis. Annals of Clinical and Translational Neurology. 2014; 1: 423-432.

[71] Ansari TL, Derakshan N. The neural correlates of cognitive effort in anxiety: Effects on processing efficiency. Biological Psychology. 2011; 86: 337-348. 